\title{
STUDIES ON A NOVEL RECOMBINANT LIPASE A FROM BACILLUS SUBTILIS FS2
}

\author{
Phung Thu Nguyet, Nguyen Hong Thanh, Truong Nam Hai* \\ Institute of Biotechnology, Vietnamese Academy of Science and Technology, \\ 18 Hoang Quoc Viet Rd., Cau Giay Dist., Hanoi, Vietnam \\ Jan-Christer Janson \\ Department of Surface Biotechnology, Biomedical Center, \\ Uppsala University, Uppsala, Sweden
}

Received 09 December 2007

\begin{abstract}
A strain of Bacillus subtilis FS2 isolated from a traditional fish source in Vietnam has been shown to produce an enzyme possessing both a gelatinase and a lipase A (LipA) activity when tested on agar plates containing $0.1 \%$ tributyrin and $0.3 \%$ gelatine. A gene encoding LipA was isolated and expressed in E. coli BL21 (DE3). The enzyme has got an apparent $M \mathrm{r}$ of $24 \mathrm{kDa}$, a pI of 9.2 and was purified to electrophoretical homogeneity by affinity chromatography. Both the lipase and the gelatinase showed optimum activity at $\mathrm{pH} 10$ and at $30^{\circ} \mathrm{C}$, with highest activity in the range $25-37^{\circ} \mathrm{C}$. The metal ions $\mathrm{Fe}^{++}, \mathrm{Co}^{++}$and $\mathrm{Mn}^{++}$at $10 \mathrm{mM}$ had strong inhibitory effect on both activities whereas $\mathrm{Ca}^{++}$had no effect and $\mathrm{Zn}^{++}$had a slight enhancing effect. The specific enzymatic activities obtained were $19814 \mathrm{U} / \mathrm{mg}$ for the lipase and 1245 $\mathrm{U} / \mathrm{mg}$ for the gelatinase.
\end{abstract}

Keywords: Bacillus subtilis FS2, lipase, gelatinase, enzyme activity, recombinant LipA

\section{INTRODUCTION}

Lipase (triacylglycerol acylhydrolase EC 3.1.1.3) is a diverse group of enzymes that catalyze the hydrolysis and transesterification of triacylglycerols, showing activity on a great variety of substrates. The commercial use of lipase comprises different industrial applications such as the production of free fatty acids, inter-esterification of oils, fats and synthesis of esters or peptides. An large number of bacterial lipases have been identified and many of these have been biochemically characterized [3]. Although a wide range of lipases are available, the interest in novel bacterial lipases is still high in order to create new applications.

In Southeast Asia, fish sauce is one of many traditional fermented foods providing important nutrients. Microorganisms isolated from fermentative products have an important potential in producing useful industrial and food enzymes such as amylases, proteases, lipases, collagenases or gelatinases etc. A strain of the gram-positive bacterium B. subtilis FS2 was isolated from Vietnamese fish sauce and its gelatinase activity was detected on agar plates containing $0.3 \%$

\footnotetext{
${ }^{*}$ Corresponding author e-mail: tnhai@hn.vnn.vn
} 
gelatine. During the isolation of the gene encoding for the gelatinase from a genomic library of B. subtilis FS2, a DNA fragment of $3.2 \mathrm{~kb}$ containing 4 open reading frames (ORFs) was discovered, in which an ORF of $600 \mathrm{bp}$ encoding for a LipA was found [7]. However, using DNA fragment deletions to find out a span of the structural gene, we discovered incidentally that the ORF encoding for the LipA was in fact also responsible for the gelatinase activity. LipA composes 181 amino acids and has got the consensus sequence Ala-Xaa-Ser-Xaa-Gly in the active site with maximum activity at $\mathrm{pH} 10$ and with a maximum stability reaching up to $\mathrm{pH} 12$.

In the past few years, many enzymes have been identified which catalyze more than one chemical transformation and this feature is called catalytic promiscuity [4]. In order to identify the reason why LipA of $B$. subtilis FS2 has got the promiscuous ability to hydrolyze also gelatine, we focused our investigation on the cloning, expression and purification of recombinant LipA from $B$. subtilis FS2 and a preliminary investigation of the properties of this novel lipase. Although there are previous reports regarding characterization for a number of Bacillus lipases, this is the first time a thorough study of the biochemical properties of a recombinant LipA (rLipA) from B. subtilis FS2 has been published.

\section{MATERIAL AND METHODS}

\subsection{Material}

Restriction enzymes, $\mathrm{T}_{4}$ ligase and Tag-DNA polymerase were purchased from Biolabs Co., UK. Collagen substrate (bovine achilles tendon, type 1) and tributyrin were from Sigma and other chemicals were of analytical grade.

\subsection{Bacterial strains, plasmid}

The B. subtilis FS2 strain was obtained from the Institute of Food Technology-Biotechnology, Ha Noi University of Technology (Vietnam), and was used as a primary material for the isolation of the LipA gene. Plasmid pBluescriptSK (+), pUC18 (Fermentas ,USA) and E. coli DH5 $\alpha$ strain were used for cloning and construction a genomic library. Plasmid pET22a $(+)$ and E. coli BL21 (DE3) strain were used for protein expression.

\subsection{Recombinant DNA-techniques}

Genomic DNA of B. subtilis FS2 was isolated according to a previously published procedure [10]. Plasmid DNA was isolated using the QIAprep Spin Miniprep Kit (QIAGEN, USA). Extraction of chromosomal DNA and plasmid from agarose gels were done with the QIAquick ${ }^{\mathrm{R}}$ Gel Extraction kit 250 (QIAGEN, USA).

Partial deletion of genomic DNA from B. subtilis FS2 with Sau3A 1 and isolation of 4-7 kb fragments were carried out as described in [7]. These DNA fragments were ligated into vector pUC18 to construct a genomic library in E. coli DH5 $\alpha$.

\subsection{Expression screening}

The tributyrin diffusion agar method was carried out according to [8]. Transformation harboring a plasmid containing the LipA gene when expressed, leads to the formation of a clear zone around the colony. The medium for screening gelatinase-producing transformations contains LB medium and a $0.3 \%$ gelatine substrate. The gelatinase activity appearing in the LB-collagen plate produced small transparent areas around the affected colonies. The gelatinase activity can be visualized unambiguously after being kept over two days in a freezer. 


\subsection{Sub-cloning of the LipA gene}

The LipA gene of 600 bp without the signal peptide sequence was amplified by PCR using specific primers. The isolated DNA fragment was cloned in the correct reading frame into the pBluescriptSK (+) vector followed by transfer of the LipA gene into the pET22a (+) expression vector (Novagen). The resulting plasmid was named pET-LipA.

\subsection{Expression and purification of rLipA}

For the expression of His-tagged rLipA, the plasmid pET-LipA was transformed in E. coli BL21 (DE3). The expression was induced by adding $1 \mathrm{mM}$ IPTG to the culture when grown to an $\mathrm{A}_{600}$ of 0.4. The cells were resuspended in buffer and disrupted by sonication and the lysate was clarified by $30 \mathrm{~min}$ centrifugation at $15000 \mathrm{rpm}$ at $4^{0} \mathrm{C}$. The supernatant was applied to a Histrap affinity column packed with Nickel-saturated Chelating Sepharose High Performance according to instructions recommended by the manufacturer. The purified rLipA was applied to the HiPrep ${ }^{\mathrm{TM}}$ Desalting 26/10 column (GE Healthcare Biosciences, Uppsala, Sweden) equilibrated in sodium phosphate buffer before being subjected to enzymatic activity assays. Active purified fractions of LipA were analysed by gradient SDS-PAGE (8-25\%) using a Phast System (GE Healthcare Biosciences, Uppsala, Sweden)

\subsection{Enzymatic activity}

The lipase activity of the purified rLipA was monitored using the Fluorescence Lipase Assay Kit (M0612, MGT-Inc, USA) DPG (1,2-dioleoyl-3 pyrenedecanoyl-rac-glycerol) as substrate. Upon cleavage, the fluorescent fatty acid PDA is released and the activity is easily obtained by measuring the absorption at 340/470 $\mathrm{nm}$ (exitation/emission).

The gelatinase activity was determined by measuring the release of the fluorescent conjugate DQ from a gelatine substrate in a reaction using the EnzChek Gelatinase/ Collagenase assay kit (Molecular Probes, USA) and a microplate fluorescence reader. The digested products from the DQgelatine substrate have got maximal absorption at $495 \mathrm{~nm}$ and emission at $515 \mathrm{~nm}$, respectively.

\subsection{Determination of temperature, $\mathrm{pH}$ and inhibitor effects on the lipase/gelatinase activity}

The effect of temperature on the lipase activity was determined by incubating aliquots of pure lipase solution in $0.1 \mathrm{M}$ glycine, $\mathrm{pH} 9.5$ for $30 \mathrm{~min}$ at various temperatures. The $\mathrm{pH}$ optimum was determined using a pH-stat assay with the fluorescent DPG substrate. The influence of various inhibitors on the lipase activity was determined by incubation of the enzyme for 60 min at $30^{\circ} \mathrm{C}$ in glycine buffer $\mathrm{pH} 9.5$, containing the reagents to be investigated. The inhibitor concentration was $0.01 \mathrm{M}$ in the incubation mixture.

The effect of temperature on the gelatinase activity was determined by incubating aliquots of pure lipase solution in $0.5 \mathrm{M}$ Tris- $\mathrm{Cl}$ buffer, $\mathrm{pH} 7.6$ for $30 \mathrm{~min}$ at various temperatures. Gelatinase activity was measured at different $\mathrm{pH}$ by the $\mathrm{pH}$-stat assay with fluorescence DQ gelatine substrate. The influence of various inhibitors on the gelatinase activity was determined by incubation of the enzyme for $60 \mathrm{~min}$ at $30^{\circ} \mathrm{C}$ in reaction buffer $\mathrm{pH} \mathrm{7.6,} \mathrm{containing} \mathrm{the}$ reagents to be determined. The inhibitor concentration was $0.01 \mathrm{M}$ in the incubation mixture.

\section{RESULTS AND DISCUSSION}

\subsection{Construction of genomic DNA of $B$. subtilis FS2}

B. subtilis FS2's DNA was partially digested with Sau3A I into small fragments of 4 to $7 \mathrm{~kb}$. 
These DNA fragments were inserted into the BamH I site of pUC18 and the ligated DNA was transformed into $E$. coli $\mathrm{DH} 5 \alpha$ cells to produce a genomic library. The transformed cells were spread on LB plates containing $0.3 \%$ gelatine and Ampicillin $(100 \mu \mathrm{g} / \mathrm{ml})$. Among the around 10,000 transformants obtained, one colony formed a clear zone on the LB plates. A $3.2 \mathrm{~kb}$ DNA insert responsible for the gelatinase expression was found in the recombinant plasmid. This DNA fragment was sequenced and shown to consist of 4 ORFs (600 bp, $365 \mathrm{bp}, 869 \mathrm{bp}$ and 749 bp) of which the ORF encoding for LipA was located at the 5' end with a length of $600 \mathrm{bp}$. The three other ORFs are encoding for unknown functional proteins. By partial deletion of a $3.2 \mathrm{~kb}$ DNA fragment by restriction enzymes, we accidentally discovered that transformants containing the ORF encoding LipA produced hydrolysis haloes around colonies when spread on agar plates containing $1 \%$ tributyrin and $0.3 \%$ gelatine. This result indicated that LipA might degrade both tributyrin and gelatine.

The LipA gene was cloned in E. coli DH5 $\alpha$ cells and a DNA sequence of 600 bp was determined. The sequence of the preprotein contains 212 amino acids in which 31 amino acids belong to a signal peptide, indicating their extracellular location. The deduced sequence of the mature LipA contains 181 amino acids.

\subsection{Purification of the expressed LipA}

rLipA was successfully expressed in E. coli BL21 (DE3) cells. The isolation of rLipA started with disintegration of the cells by sonification followed by centrifugation. To increase the efficiency of the cell lysis process, lysozyme at a final concentration of $100 \mu \mathrm{g} / \mathrm{ml}$ was added followed by incubation at $-20^{\circ} \mathrm{C}$ overnight. In the next step, affinity chromatography was used on a Hi-Trap affinity column equilibrated in $50 \mathrm{mM}$ sodium phosphate buffer, $\mathrm{pH}$ 7.4. The purified LipA was eluted from column with the sodium phosphate buffer containing $600 \mathrm{mM}$ imidazole.

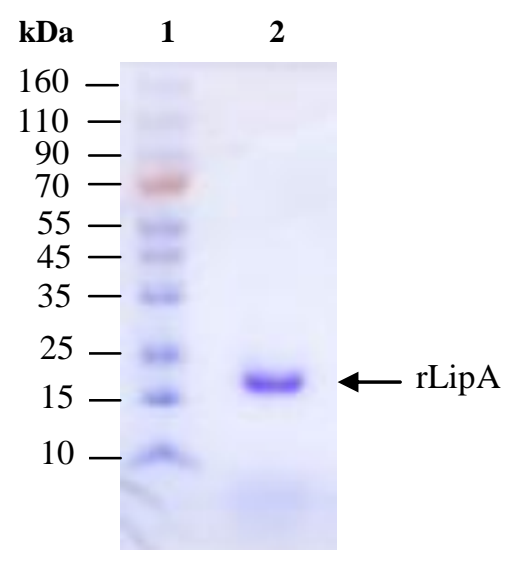

Fig. 2: SDS-PAGE of purified rLipA. Lane 1: Pre-staining marker, Lane 2: Purified rLipA.

SDS-PAGE showed a single protein band corresponding to a molecular weight approximately of $24 \mathrm{kDa}$ in the fraction with $600 \mathrm{mM}$ imidazole (Fig. 2). The enzyme was applied to a HiPrep $^{\mathrm{TM}}$ Desalting 26/10 column before subjected to enzymatic activity assay.

\subsection{Lip A specific activity}

The lipase and gelatinase activities of Lip A from B. subtilis FS2 were demonstrated on agar plates containing $0.1 \%$ tributyrin and $0.3 \%$ gelatine (data is not shown). The specific activity of 
the lipase was determined using the fluorescent substrate 1,2-dioleoyl-3-pyrenedecanoyl-racglycerol (DPG). One unit of activity was defined as the amount of enzyme that released $1 \mu \mathrm{mol}$ DPG substrate per minute under the assay condition used. The specific activity of LipA was determined to be $19814 \mathrm{U} / \mathrm{mg}$. Due to the fact that B. subtilis LipA is lacking the hydrophobic lid covering the active site of typical lipases, LipA has got asolvent-exposed active site.

For the gelatinase, the specific activity of LipA was determined using the DQ gelatine substrate. 1 enzyme unit was defined as the amount of enzyme that catalyzes the reaction of $1 \mu \mathrm{mol}$ DQgelatine substrate per minute under standard conditions and the specific activity was determined to be $1245 \mathrm{U} / \mathrm{mg}$.

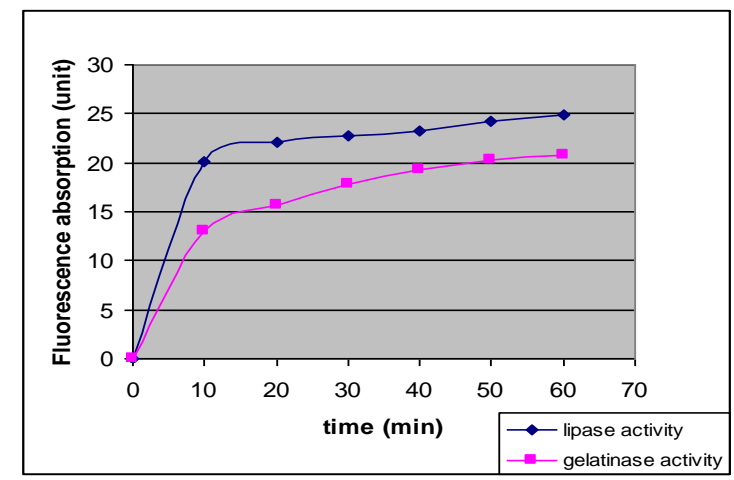

Fig. 3: Plotting of fluorescence and time for lipase (•) and gelatinase ( $\boldsymbol{\square})$ determined by continuously recording the concentration of fluorescent conjugate released at $10 \mathrm{~min}$ intervals for 60 mins.

A possible explanation to the gelatinase activity of this lipase might be hidden in a temporary stabilization of the transition state. A lipase normally cleaves a C-O bond in a triacylglycerides and a gelatinase, in analogy with serine proteases is able to hydrolyze a C-N bond. Although C$\mathrm{N}$ and $\mathrm{C}-\mathrm{O}$ bond hydrolysis differ chemically, the catalytic mechanism must be very similar since serine proteases have the same catalytic triad as lipases, i.e. composed of Ser-His-Asp (or Glu). Our hypothesis is that the Bacillus lipase has evolved a similar stabilization of a tetrahedral intermediary state in the catalytic mechanism of the enzyme.

\subsection{Enzyme characterization}

To evaluate the effect of various factors on the lipase and gelatinase activities, investigations were performed under a series of different conditions regarding $\mathrm{pH}$, temperature and metal ions. For the lipase, the purified rLipA showed maximum activity at $\mathrm{pH} 10$ and rapidly decreasing at $\mathrm{pH}$ values below 7. Compared to previous reports [5] these data showed that the LipA of $B$. subtilis 168 can be operated at an optimal $\mathrm{pH}$ of 10 and is remarkably stable after 60 minutes at this $\mathrm{pH}$. This is why LipA of $B$. subtilis can be called an alkaline lipase.

The effect of $\mathrm{pH}$ on the gelatinase activity was similar to that on the lipase activity with maximum activity at $\mathrm{pH} 10$. Fig 4 shows that lipase and gelatinase have similar $\mathrm{pH}$-dependencies but that the effect on the lipase is more pronounced than that on the gelatinase.

The rLipA exhibited a temperature maximum at about $30^{\circ} \mathrm{C}$ for both lipase and gelatinase activities and stability for at least $60 \mathrm{~min}$ in the range $25^{\circ} \mathrm{C}$ to $45^{\circ} \mathrm{C}$ for the gelatinase activity (Fig. 5). In a previous study, the gelatinase of B. subtilis $\mathrm{CN} 2$ showed a temperature maximum at $50^{\circ} \mathrm{C}$ and a relatively high activity over a broad temperature range $\left(30-60^{\circ} \mathrm{C}\right)$ [12]. These data 
show that the thermo stability for the gelatinase of the rLipA from B. subtilis FS2 differs significantly from that of $B$. subtilis $\mathrm{CN} 2$.

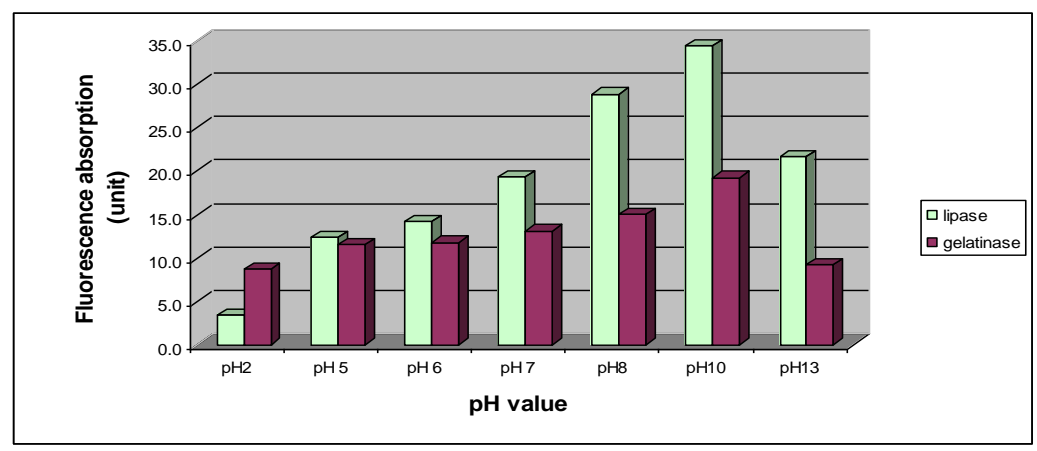

Fig. 4: Effect of $p H$ on lipase and gelatinase activity.

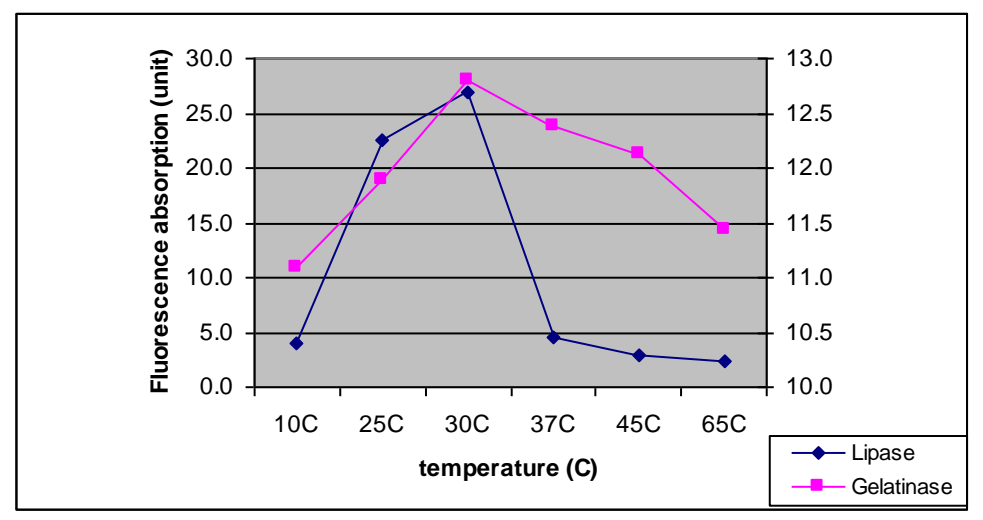

Fig. 5: Effect of temperature on the lipase and gelatinase activitiy.

Both the lipase and the gelatinase activities are strongly inhibited by $\mathrm{Fe}^{++}, \mathrm{Co}^{++}$and $\mathrm{Mn}^{++}$at 10 $\mathrm{mM}$ concentration. The effect of metal ions on the lipase activity probably results from a change in the solubility and the behaviour of the ionized fatty acids at interfaces.

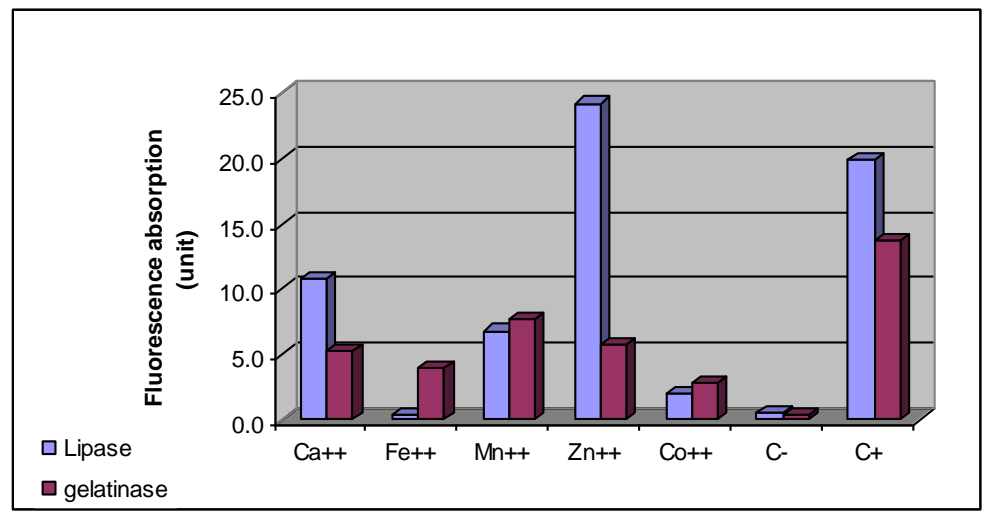

Fig. 6: Effect of metal ions on the lipase and gelatinase activity. Control -: reaction without DQ gelatine substrate. Control +: reaction contain DQ gelatine substrate. 
Alternatively, the metal ions can change the catalytic properties of the enzyme itself. A similar inhibitory effect of the $\mathrm{Fe}^{++}$ion has been reported previously [9]. However, $\mathrm{Ca}^{++}$ions did not stimulate the lipase activity (Fig 6) but $\mathrm{Zn}^{++}$ion has a slight enhancing effect. These data do not confirm those obtained by Lesuisse et al [5] who showed that $\mathrm{Ca}^{++}$ion had stimulatory effects on the lipase from $B$. subtilis 168 . In that study no other metal ions tested were able to replace $\mathrm{Ca}^{++}$with full retention of activity. This result suggests that the effect of these inhibitors are directly involved in the catalytic site and that the catalytic residues of rLipA from $B$. subtilis FS2 differ from that of LipA from B. subtilis 168. To confirm this hypothesis, a series of experiments involving mutation of residues in the catalytic site should be investigated.

\section{ACKNOWLEDGEMENT}

This work was supported by the Vietnam-Sweden Research Cooperation Program SIDA/SAREC with the project "Production of recombinant protein for agricultural and medical use", code VS/BT3.

\section{REFERENCES}

1. Jaeger, K.E., Dijkstra, B.W., and Reetz, M.T. (1999), Bacterial catalysts: molecular biology, three dimension structure and biological application of Lipase, Annual Rev Microbial 53, pp. 315-351.

2. Jaeger, K.E. and Eggert, T. (2002), Lipases for biotechnology. Curr Opin Biotechnol 13(4), pp. 390-397

3. Jaeger, K.E. and Reetz, T.M. (1998), Microbial lipases from versatile tools for biotechnology. Trends Biotechnol 16(9), pp. 396-403.

4. Kourist, R., Bartsch, S., Fransson, L., Hult, K., and Bornscheuer, U.T. (2008), Understanding promiscuous amidase activity of an esterase from Bacillus subtilis, Chembiochem 9(1), pp. 67-9.

5. Lesuisse, E., Schanck, K., and Colson, C. (1993), Purification and preliminary characterization of the extracellular lipase of Bacillus subtilis 168, an extremely basic $\mathrm{pH}$ tolerant enzyme, Eur J Biochem 216, 155-160.

6. Nguyen Hong Thanh, Phung Thu Nguyet, Truong Nam Hai (2007), Expression of gene encoding lipase (LipA) from Bacillus subtilis FS2, J Biotech 5(1), pp. 41-46.

7. Phung Thu Nguyet, Tran Minh Tri, Nguyen Hong Thanh, To Kim Anh, Truong Nam Hai (2005) Cloning and expression of gene encoding for collagenase from B. subtilis FS-2. Proceeding of International Vietnam - Korea Symposium:16-21.

8. Rani, G., Pooja, R., Namita, G., Sapna, B (2003), Lipase assays for conventional and molecular screening: an overview, Biotechnol Appl Biochem. 37, pp. 63-71.

9. Rhohit, S., Yusuf, C., Uttam. CB (2001), Production, purification, characterization and applications of lipases, Biotechnol Adv 19, pp. 627-662.

10. Sambrock, J. et al. (1989) Molecular Cloning: A Laboratory Manual. 2d Edition. Cold Spring, Harbor Press, Cold Spring Harbor.

11. Throsten, E., Gertie, P., Gaelle, P, Isabelle, D., Robert, V., Bauke, W., Dijksra, Karl. E.J. (2002), Biochemical properties and three dimensional structure of two extracellular lipolytic enzymes from Bacillus subtilis, Biointerfaces 26, pp. 37-46. 
12. Uchida, H., Kondo, D., Yamashita, S., Tanakanm T., Tran, L.H., Nagano, H., Uwajima, T. (2004), Purification and properties of protease produced by Bacillus subtilis CN2 from Vietnamese fish sauce, World J Microbiol Biotech 20, pp. 579-582. 